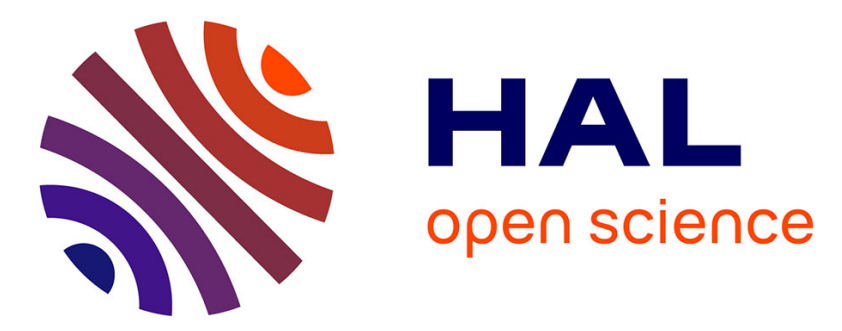

\title{
Utilité sociale des représentations intergroupes de sexe. Domination masculine, contexte professionnel et discrimination positive
}

\author{
Pascal Moliner, Fabio Lorenzi-Cioldi, Elise Vinet
}

\section{To cite this version:}

Pascal Moliner, Fabio Lorenzi-Cioldi, Elise Vinet. Utilité sociale des représentations intergroupes de sexe. Domination masculine, contexte professionnel et discrimination positive. Les cahiers Internationaux de Psychologie Sociale, 2009, 83, pp.25 - 44. 10.3917/cips.083.0025 . halshs-01561806

\section{HAL Id: halshs-01561806 \\ https://shs.hal.science/halshs-01561806}

Submitted on 13 Jul 2017

HAL is a multi-disciplinary open access archive for the deposit and dissemination of scientific research documents, whether they are published or not. The documents may come from teaching and research institutions in France or abroad, or from public or private research centers.
L'archive ouverte pluridisciplinaire HAL, est destinée au dépôt et à la diffusion de documents scientifiques de niveau recherche, publiés ou non, émanant des établissements d'enseignement et de recherche français ou étrangers, des laboratoires publics ou privés. 


\section{UTILITÉ SOCIALE DES REPRÉSENTATIONS INTERGROUPES DE SEXE. DOMINATION MASCULINE, CONTEXTE PROFESSIONNEL ET DISCRIMINATION POSITIVE}

Pascal Moliner, Fabio Lorenzi-Cioldi, Élise Vinet

Presses universitaires de Liège | «es Cahiers Internationaux de Psychologie

Sociale »

2009/3 Numéro 83 | pages 25 à 44

ISSN 0777-0707

Article disponible en ligne à l'adresse :

http://www.cairn.info/revue-les-cahiers-internationaux-de-psychologiesociale-2009-3-page-25.htm

\section{Pour citer cet article :}

Pascal Moliner et al., «Utilité sociale des représentations intergroupes de sexe. Domination masculine, contexte professionnel et discrimination positive », Les Cahiers Internationaux de Psychologie Sociale 2009/3 (Numéro 83), p. 25-44. DOI 10.3917/cips.083.0025

Distribution électronique Cairn.info pour Presses universitaires de Liège.

(C) Presses universitaires de Liège. Tous droits réservés pour tous pays.

La reproduction ou représentation de cet article, notamment par photocopie, n'est autorisée que dans les limites des conditions générales d'utilisation du site ou, le cas échéant, des conditions générales de la licence souscrite par votre établissement. Toute autre reproduction ou représentation, en tout ou partie, sous quelque forme et de quelque manière que ce soit, est interdite sauf accord préalable et écrit de l'éditeur, en dehors des cas prévus par la législation en vigueur en France. Il est précisé que son stockage dans une base de données est également interdit. 


\section{6}

Utilité sociale des représentations intergroupes de sexe. Domination masculine, contexte professionnel et discrimination positive

\section{Social utility of intergroup} gender representations:

Male domination, occupational context, and affirmative action

Pascal MOLINER*, Fabio LORENZI-CIOLDI** et Élise VINET*

* Laboratoire de Psychologie, Université Paul Valéry, Montpellier, France

** Groupe de recherche sur les relations intergroupes et les représentations sociales, Université de Genève, Genève, Suisse 
Dans trois expériences les participants lisent les descriptions d'un homme et d'une femme, présentés dans différents contextes (familial vs professionnel ; position professionnelle dominante masculine vs féminine; promotion professionnelle de la femme motivée par ses compétences vs une mesure de discrimination positive). Ils attribuent ensuite des comportements et des traits de personnalité, différenciés selon leur stéréotypie sexuelle, à chacune des deux personnes. Les résultats montrent que le contexte professionnel conduit à une accentuation des attributions stéréotypées. On constate aussi qu'une position de domination confère à son occupant, homme ou femme, une stéréotypie masculine. Toutefois, cette stéréotypie s'atténue pour le personnage féminin lorsque son accès à la position de domination résulte d'une mesure de discrimination positive.

Représentation intergroupes - stéréotype - sexe - hiérarchie - discrimination positive.
In three experiments, participants read descriptions of a man and a woman, which were presented in different contexts (home vs work context; male vs female professional dominant position professional promotion of the woman due to her competence vs. to an affirmative action policy). They then attributed to each of the two people behaviors and personality traits that were differentiated according to gender stereotypes. The results showed that participants accentuated stereotypical attributions in the work context, and that a high-status position led to masculine attributions to the person holding the position, whether that person was a man or a woman. However, attribution of masculinity to the woman was reduced when the reason for her promotion to the high-status position was based on an affirmative action policy.

Intergroup representations - stereotype gender - hierarchy - affirmative action.

La correspondance pour cet article doit être adressée à Pascal Moliner, Laboratoire de psychologie, Université Paul Valéry, route de Mende, 34199 Montpellier CEDEX 5, France. Courriel : <pascal.moliner@univ-montp3.fr>. Contribution des auteurs : Pascal Moliner (conception étude 1, recueil des données et rédaction), Fabio LorenziCioldi (conception étude 3 et rédaction) et Élise Vinet (conception étude 2). 
Selon les promoteurs de la notion de représentation intergroupes (Doise, 1973 ; Deschamps, 1973), celles-ci sont le fruit d'un processus de catégorisation sociale (Tajfel, 1972), " qui rend compte de la division entre le nous et le eux » (Deschamps, 1973, p. 710). Les représentations émergent et évoluent « au travers des jugements portés par des groupes sur d'autres, jugements eux-mêmes déterminés par la nature des rapports entre ces groupes » (ibid., p. 715). Elles remplissent deux fonctions essentielles. En premier lieu, elles permettent aux membres d'un groupe de justifier leurs comportements à l'égard des membres d'un autre groupe. En second lieu, elles leur permettent d'anticiper les interactions avec les membres de l'exogroupe et, de ce fait, induisent certains comportements (Doise, 1969, 1973). Dans la présente recherche, nous avançons l'idée que les représentations intergroupes sont une forme particulière de représentations sociales. À ce titre, elles remplissent une fonction d'explication des conduites des membres de l'endogroupe et de ceux de l'exogroupe. Ainsi, les représentations intergroupes permettent d'anticiper des comportements et de les expliquer. Dans le cas particulier des relations intergroupes asymétriques, ces anticipations et ces explications amènent les membres du groupe de haut statut à justifier leur position de domination, tandis qu'elles amènent les membres du groupe de bas statut à rationaliser leur position de subordination (e.g., Jost et Burgess, 2000 ; Jost et Kay, 2005). Dès lors, on peut penser que $c^{\prime}$ est dans les contextes sociaux où l'asymétrie des rapports entre les groupes est la plus prégnante que les individus mobiliseront le plus leurs représentations intergroupes.

\section{Représentations intergroupes et représentations sociales}

Les représentations sociales sont des modalités particulières de connaissance, proches du savoir naïf ou du sens commun, qui permettent aux groupes et aux individus de comprendre, d'interpréter et de donner du sens à leur environnement social (Kelley, 1992 ; Moscovici, 1961). Selon l'approche dite «structurale» (Abric, 1976, 1994), les éléments qui constituent ces représentations se distinguent en fonction de leur statut " central » ou " périphérique ». Les éléments centraux, consensuels et stables, déterminent la signification des autres éléments, et finalement la signification globale d'une représentation sociale. Les éléments périphériques, peu consensuels et davantage liés aux contextes, constituent les traces des expériences individuelles relatives à l'objet de représentation (Flament, 2001). Si I'on admet donc que les représentations intergroupes sont une forme particulière de représentation sociale, il est raisonnable de supposer qu'elle remplissent la fonction de connaissance qui est habituellement attribuée à ces dernières (Abric, 1994). Les représentations intergroupes contiendraient ainsi des éléments de description et $d^{\prime}$ explication permettant aux individus qui en sont porteurs de comprendre et d'expliquer les comportements des membres de leur propre groupe et de l'exogroupe.

Plusieurs recherches récentes suggèrent que les représentations intergroupes contiennent bien des éléments explicatifs. Par exemple, Moliner et Gutermann (2004) ont constaté, auprès d'une population de bénévoles impliqués dans des actions de prévention de la délinquance, la présence de croyances explicatives des 
comportements délinquants. Ainsi, $63 \%$ des sujets interrogés estiment que les comportements délinquants ou déviants s'expliquent par le fait que ceux qui en sont les auteurs «ne sont pas intéressés par le travail ». Dans une autre recherche (Vinet et Moliner, 2006), les participants indiquaient les traits qui " expliquent le mieux » les comportements des hommes et des femmes en général. Les résultats mettent en évidence un fort consensus entre les participants. Par exemple, hommes et femmes se rejoignent pour estimer que les comportements des femmes s'expliquent par leur « douceur » $(100 \%$ des femmes et $83 \%$ des hommes), leur « sensibilité » $(94 \%$ et $89 \%$ ) et leur " affectivité » (88\% et $89 \%$ ). De même, les participants expliquent le comportement des hommes par leur « force » $(88 \%$ des femmes et $85 \%$ des hommes), leur « domination » (82\% et $62 \%)$ et leur « fierté » $(94 \%$ et $90 \%)$.

L'approche structurale appliquée à l'étude des représentations intergroupes conduit ainsi à l'idée de stéréotype. En effet, l'apparition de réponses consensuelles, comme I'illustrent les exemples mentionnés ci-dessus, s'apparente à un stéréotype amenant les individus à surestimer les ressemblances entre les membres d'une même catégorie (Tajfel et Wilkes, 1963). Lorsqu'une représentation intergroupes est étudiée à travers l'approche structurale, éléments stéréotypiques et éléments centraux se confondent (Vidal, 2003).

\section{L'utilité sociale des représentations de sexe}

Les rapports de sexe demeurent, dans nos sociétés, caractérisés par une profonde asymétrie. De nombreux indicateurs socio-économiques (p. ex., l'occupation de postes décisionnaires, le salaire, la représentation politique) signalent que la plupart des positions dominantes dans les hiérarchies notamment professionnelles des positions qui confèrent du pouvoir de décision restent largement inaccessibles aux femmes (Bihr et Pfefferkorn, 2002). Ces faits se répercutent sur les représentations de sexe (Diekman et Eagly, 2000 ; Lorenzi-Cioldi, 2002).

Nous avons vu qu'il existe un consensus sur les contenus de ces représentations. Ce consensus s'exprime le long de dimensions qui ont été dénommées « instrumental » (pôle masculin) vs. " expressif » (pôle féminin), ou encore " agentique vs. communial », "personnologique vs. collectif », etc. (cf. Lorenzi-Cioldi, 1994). Or une contribution de l'approche des " principes organisateurs » à l'étude des représentations intergroupes (Doise, 2005) a été de montrer que sous ce consensus se cachent de profondes variations pouvant être captées par plusieurs sortes d'analyses multivariées (Doise, Clémence et Lorenzi-Cioldi, 1992). C'est ainsi que de fortes variations dans les représentations persistent même lorsque l'observateur est amené à constater qu'hommes et femmes se comportent (souvent) de la même manière. Par exemple, Lorenzi-Cioldi (1994) constate qu'un comportement donné est expliqué de manière favorable lorsqu'il est effectué par l'homme (il dénote ainsi, par exemple, de l'assurance en soi, de la franchise, etc.), et défavorable lorsqu'il est effectué par la femme (dénotant de l'arrogance, de l'irritation, etc.). Aussi, un comportement fortement normatif dans les sociétés occidentales, comme l'est une manière d'agir " indépendante » et "individualiste», est jugé à l'aide de traits comme " se suffit à soi-même », " agressif » et " dominateur » s'il est déployé par 
un homme, mais à l'aide de traits comme " réservée », " timide » et " imprévisible » s'il est déployé par une femme.

Plus généralement, les contenus des représentations de sexe reflètent les rapports de domination / subordination dans lesquels sont impliqués les unes et les autres. Les concepts de groupes « collection » et « agrégat » ont été proposés par LorenziCioldi $(2002,2006)$ pour rendre compte de la structure et des contenus de ces différences. Ainsi, les membres d'un groupe socialement dominé se perçoivent et sont perçus par autrui comme un ensemble de personnes relativement indifférenciées les unes des autres, par rapport aux membres du groupe dominant qui se perçoivent et sont perçus comme des personnes différentes les unes des autres, comme des êtres uniques et distingués, des " personnalités » qui n'ont point besoin d'une appartenance commune pour se définir.

Or des recherches récentes tendent à montrer que dominants et dominés partagent ces représentations différenciées des uns et des autres (e.g., Chatard, LorenziCioldi, et Guimond, 2007 ; Hoffman et Hurst, 1990 ; Jost et Kay, 2005 ; Sidanius et Pratto, 1999). Partant, on peut supposer que ces représentations leur permettent non seulement de comprendre, mais également de justifier et de rationaliser leurs positions de domination et de subordination, contribuant ainsi à perpétuer le statu quo de la relation sociale asymétrique. Dans leur modèle de la « justification du système », Jost et Banaji (1994) avancent précisément l'idée selon laquelle les stéréotypes permettent aux individus une meilleure acceptation de leur situation sociale, qu'elle soit de domination ou de subordination ( $c f$. aussi Jost, Banaji, et Nosek, 2004). Les stéréotypes décrivent, expliquent et justifient les hiérarchies de statuts. En d'autres termes, ils amènent dominants et dominés à coopérer en vue de la défense du statut quo.

Dans cette perspective, $c^{\prime}$ est dans des contextes sociaux où les rapports de domination sont les plus prégnants que les individus devraient mobiliser le plus fortement les représentations intergroupes. Cette hypothèse a été mise à l'épreuve dans trois études expérimentales qui impliquent le critère de catégorisation du sexe.

\section{Vue d'ensemble des études}

Trois facteurs ont été manipulés à travers des procédures expérimentales : la position statutaire d'un homme et d'une femme (dominante vs. subordonnée), le contexte dans lequel se déploie cette relation de statutaire (familial vs. professionnel), et la cause de l'asymétrie statutaire (compétence vs. action positive). L'impact de ces inductions a été mesuré en observant la manière dont les participants attribuent des comportements et des traits de personnalité différenciés selon leur stéréotypie sexuelle à chacune des deux personnes.

L'étude introductive compare le contexte familial au contexte professionnel, en insistant toutefois sur l'égalité des deux personnes en termes de plusieurs caractéristiques habituellement liées au statut social (diplômes, compétences, revenus). On devrait observer ici, en conformité avec les explications classiques des stéréotypes 
de sexe (e.g., Eagly, 1987), que le contexte professionnel, en ravivant le sentiment de l'inégalité sociale de l'homme et de la femme, produit davantage de stéréotypie sexuelle que le contexte familial. La seconde étude prend en compte uniquement le contexte professionnel mais varie la position statutaire relative de l'homme et de la femme. À une situation « attendue » où l'homme occupe un meilleur statut professionnel que la femme, on opposera une situation « inattendue » présentant la situation opposée. L'idée de dominance étant associée au stéréotype de la masculinité, en particulier dans un environnement professionnel, on s'attend que le personnage qui occupe la position de haut statut, qu'il soit homme ou femme, sera perçu comme plus masculin que le personnage qui occupe la position de bas statut. Cette attente ne surprend guère pour ce qui est de l'homme, pour lequel la position statutaire suggérée par le stéréotype et celle manipulée expérimentalement sont congruentes. Pour ce qui est de la femme, en revanche, la vérification de cette attente permettrait de mieux comprendre pourquoi, comme le montre la littérature psychosociale, la femme qui réalise une mobilité professionnelle est souvent sommée de « renier son sexe » pour adopter des comportements, voire des traits de personnalité, plus masculins (e.g., Ellemers, 2001). L'étude 3 manipule un facteur qui devrait moduler ce conflit identitaire chez la femme en milieu professionnel. À une femme promue à un statut supérieur à celui de l'homme grâce à ses compétences (et donc conformément au critère méritocratique à l'œuvre dans un contexte de domination masculine), on opposera une femme qui accède à ce statut en bénéficiant d'une mesure positive. On s'attend que le conflit identitaire, pour la cible féminine, soit moins prononcé dans cette dernière situation.

\section{ÉTUDE 1 : Domination masculine en contextes privé et professionnel}

\section{Procédure}

Les participants étaient 40 étudiantes et 40 étudiants de 1 ère année de psychologie (âge moyen $=19$ ans). Les participants lisaient un court scénario dans lequel étaient décrits un homme et une femme. Ces personnages étaient présentés soit dans un contexte professionnel (PRO), soit dans un contexte privé (PRIV). En contexte PRO, le scénario énonçait que «Sophie et François travaillent dans la même entreprise où ils partagent un bureau commun. Dans leur travail, ils font équipe et sont placés sous l'autorité du même supérieur hiérarchique. Par ailleurs, en termes de diplômes et de compétences, Sophie et François sont à niveau égal ». En contexte PRIV, le scénario était ainsi formulé : "Sophie et François vivent en couple et partagent le même appartement. Dans la vie quotidienne, ils ont pris l'habitude de se répartir les tâches. Par ailleurs, en termes de diplômes et de revenus, Sophie et François sont à niveau égal ».

La tâche des participants consistait alors, dans un premier temps, à anticiper les comportements de chacun des deux personnages. Pour cela, ils disposaient d'une liste de 8 comportements, dans laquelle ils sélectionnaient 3 comportements pour François et 3 comportements pour Sophie. S'inspirant largement de l'inventaire de Bem (1974), cette liste incluait des comportements stéréotypiquement masculins 
supposant l'assurance, l'autorité, voire I'agressivité (Donner des conseils, Savoir imposer son point de vue, Provoquer les conflits quand ils sont nécessaires, et Défendre ses idées jusqu'au bout), et autant de comportements stéréotypiquement féminins supposant la capacité d'écoute, la compréhension et le caractère apaisant (Reconnaitre quand l'autre a raison, Éviter les conflits, Accepter les idées de l'autre, et Écouter l'avis de l'autre). Pour l'essentiel, ces comportements s'alignent sur les pôles « instrumental vs expressif » évoqués plus haut.

Dans un second temps, les participants expliquaient les comportements qu'ils venaient de sélectionner à l'aide d'une liste composée pour moitié de traits masculins (force, domination, et fierté) et pour moitié de traits féminins (douceur, sensibilité, et affectivité) (cf. Vinet et Moliner, 2006). Ils choisissaient d'abord trois traits pour expliquer les comportements d'un personnage, puis utilisaient la même liste pour choisir trois traits expliquant les comportements de l'autre personnage. La moitié d'entre eux jugeaient d'abord les comportements de François, I'autre moitié d'abord ceux de Sophie'.

Le plan expérimental comporte trois facteurs : 2 (sexe du personnage) x 2 (contexte : PRIV vs. PRO) x 2 (sexe du participant), le premier facteur étant en mesure répétée. L'hypothèse s'énonce sous la forme d'une interaction entre le sexe du personnage et le contexte. Le contexte PRO, par rapport au contexte PRIV, devrait introduire une plus forte prégnance du rapport asymétrique entre l'homme et la femme, et induire ainsi les participants à mobiliser plus fortement des représentations intergroupes visant à justifier ce rapport. On s'attend donc à l'émergence d'une stéréotypie sexuelle plus intense de l'homme et de la femme dans le contexte PRO que dans le contexte PRIV, aussi bien pour ce qui est des comportements sélectionnés que des explications de ces comportements.

\section{Résultats}

Afin de procéder à la vérification de notre hypothèse, nous avons calculé deux scores de stéréotypie sexuelle. Le score de comportements anticipés résulte, pour chaque personnage, de la somme des comportements masculins qui lui ont été assignés. La valeur maximale de 3 indique que le personnage a été décrit exclusivement par des comportements masculins, et la valeur minimale de 0 qu'il a $^{\prime}$ été décrit exclusivement par des comportements féminins. De manière analogue, le score d'attributions résulte de la somme des explications masculines des comportements. Lorsqu'il est égal à 3, le personnage a été jugé tout à fait masculin, et lorsqu'il est égal à 0 , il a été jugé tout à fait féminin.

Les corrélations entre ces deux scores, toutes conditions confondues, apparaissent élevées $(0,65$ concernant Sophie, et 0,69 concernant François, $p s<.01)$. Ce résultat atteste le lien entre comportements anticipés et leurs explications. Globalement, quel que soit le sexe des participants et celui du personnage, et quel que soit le contexte, I'anticipation de comportements masculins entraîne des attributions masculines, tandis que l'anticipation de comportements féminins entraîne des attributions féminines. 
L'analyse de variance (ANOVA) ne montre aucun effet simple du sexe des participants. Par ailleurs, on note que ce facteur n'interagit avec aucun des deux autres facteurs de l'analyse. En revanche, I'ANOVA met en évidence, conformément à notre hypothèse, une interaction entre le contexte et le sexe du personnage, $F(1,76)=$ $5,88, p<.02$. Le Tableau 1 présente les moyennes de ce score.

Tableau 1 : Score de comportements anticipés $(0=$ féminin ; 1,5 = autant féminin que masculin ; 3 =masculin). Les écarts-type sont indiqués entre parenthèses. Comparaison avec $1,5: * p<.05$.

\begin{tabular}{cccc}
\hline & \multicolumn{2}{c}{ Contexte } & \\
\cline { 2 - 3 } & Pro & Priv & Moyenne \\
\hline François & $1,80^{*}(0,88)$ & $1,40(0,81)$ & 1,60 \\
Sophie & $1,40(0,67)$ & $1,80^{*}(0,85)$ & 1,60 \\
Moyenne & 1,60 & 1,60 & \\
\hline
\end{tabular}

En condition $\mathrm{PRO}$, les participants tendent à anticiper davantage de comportements masculins pour François que pour Sophie, $F(1,76)=2,94, p=.09$. En condition PRIV, on observe la tendance inverse, $F(1,76)=2,94, p=.09$. La comparaison des deux contextes révèle l'imputation de plus de comportements masculins pour François en condition PRO qu'en condition PRIV, $F(1,76)=4,38, p<.05$, et de plus de comportements masculins pour Sophie en condition PRIV qu'en condition PRO, $F(1,76)=5,43, p<.05$.

Afin de déterminer la stéréotypie sexuelle de chaque personnage, on peut en outre comparer le score observé à la valeur de 1,5, qui signale un même nombre moyen d'imputations de comportements masculins et féminins. En effectuant cette comparaison dans chacune des cases du Tableau 1, on remarque qu'en condition PRO les comportements anticipés pour François sont nettement masculins, $z=2,15, p<$ .05 , tandis que les comportements anticipés pour Sophie, ne s'écartant pas significativement de la valeur centrale de l'indice, sont plus indifférenciés. La condition PRIV fait apparaître le phénomène opposé : ce sont les comportements anticipés pour Sophie qui apparaissent comme masculins, $z=2,20, p<.05$.

Pour ce qui concerne les explications de ces comportements, I'ANOva ne montre aucun effet simple du sexe des participants, ni aucune interaction avec les autres facteurs pris en considération. Le sexe du personnage produit un effet ( $c f$. Tableau 2). Les participants font davantage d'attributions masculines pour François que pour Sophie, $F(1,76)=19,88, p<.001$. L'ANOva produit également l'interaction attendue entre le contexte et le sexe du personnage, $F(1,76)=6,86, p<.02$.

En condition $\mathrm{PRO}$, les attributions pour François sont plus masculines que pour Sophie, $F(1,76)=25,48, p<.001$. De plus, la comparaison à 1,5 (indifférenciation des attributions) montre que François est perçu comme masculin, $z=5,47, p<.05$, 
et Sophie comme féminine, $z=4,39, p<.05$. En revanche, dans la condition PRIV les attributions sont similaires pour les deux personnages, et plus indifférenciées selon la stéréotypie de genre. Enfin, les participants font davantage d'attributions masculines pour François en contexte PRO qu'en contexte PRIV, $F(1,76)=9,46$, $p<.005$, mais ils font le même type d'attributions pour Sophie, quel que soit le contexte.

Tableau 2 : Score d'attribution ( $0=$ féminin ; $1,5=$ autant féminin que masculin ; $3=$ masculin). Les écarts-type sont indiqués entre parenthèses. Comparaison avec $1,5:{ }^{* *} p<.01$.

\begin{tabular}{cccc}
\hline & \multicolumn{2}{c}{ Contexte } & \\
\cline { 2 - 3 } & Pro & Priv & Moyenne \\
\hline François & $2,18^{* *}(0,78)$ & $1,55(1,04)$ & 1,86 \\
Sophie & $0,93^{* *}(0,83)$ & $1,23(1)$ & 1,08 \\
Moyenne & 1,55 & 1,39 & \\
\hline
\end{tabular}

\section{Discussion}

Les résultats de l'Étude 1 concordent avec notre attente : la mobilisation de représentations intergroupes stéréotypiques s'intensifie dans un contexte qui rend prégnante l'asymétrie du rapport des deux groupes par rapport à un contexte qui la tempère, permettant ainsi de justifier, ou de rationaliser, ce rapport. Les résultats mettent en évidence une accentuation des stéréotypes de sexe dans le contexte professionnel par rapport au contexte de vie privée. François y est en effet perçu comme plus masculin, et Sophie comme plus féminine.

Ce résultat laisse présager des mutations dans les rôles sociaux assumés par les unes et les autres dans différents contextes. Selon le modèle de Eagly (1987), les rôles sociaux dévolus aux membres d'un groupe déterminent les stéréotypes appliqués à ce groupe. Or des enquêtes récentes indiquent que la vie de couple, le partage des tâches, etc., évoluent vers plus d'égalité (du moins dans les pays occidentaux), tandis que la carrière professionnelle, notamment l'obtention de postes de responsabilité dans les entreprises, demeurent encore largement inaccessibles aux femmes (cf. par exemple, Eagly et Sczeny, 2009; Meron, Okba et Viney, 2006). Le fait que, dans notre dispositif expérimental, aucune information ne renseignait les participants sur une quelconque relation asymétrique entre l'homme et la femme, tend à corroborer l'idée selon laquelle des croyances diffuses et implicites sur les rôles qui sont considérés comme étant habituellement assumés par les hommes et les femmes suffit à dicter des anticipations de comportements et de traits de personnalité conformes à ces croyances ( $c f$. aussi Ridgeway et Erickson, 2000).

Ainsi, on peut supposer que les participants ont inféré une asymétrie statutaire sur la seule base du sexe des personnages, c'est-à-dire dans un contexte où cette asymétrie n'était pas rendue explicite, mais est socialement très répandue. Si tel est le 
cas, I'explicitation d'une relation asymétrique en faveur du personnage masculin devrait conduire les participants à produire ces mêmes perceptions. En revanche, l'explicitation d'une relation asymétrique inattendue, où le personnage féminin est en position dominante, devrait favoriser l'émergence de descriptions différentes. Cette différence peut prendre plusieurs formes, que nous allons introduire ci-après.

\section{ÉTUDE 2 : Domination masculine vs. féminine}

Le propos de cette étude était de comparer le contenu des représentations de sexe dans un contexte qui, véhiculant l'asymétrie des positions occupées par un homme et une femme en milieu professionnel, souligne cette asymétrie (homme occupant une position dominante), ou la renverse (femme occupant une position dominante). On s'attend à répliquer les résultats de l'étude précédente, mais seulement lorsque la relation asymétrique calque les rapports de sexe courants.

\section{Procédure}

Les participants étaient 20 étudiantes et 20 étudiants de $1^{\text {ère }}$ année de psychologie (âge moyen $=20$ ans). Ils étaient d'abord invités à examiner le scénario décrivant le contexte professionnel, identique à celui utilisé dans l'Étude 1, mais complété afin d'expliciter la situation de domination. En situation de domination masculine (DM), le scénario se terminait ainsi : "Pourtant, dans leur travail, ils ne sont pas sur un même pied d'égalité, et François a plus de pouvoir que Sophie ». En situation de domination féminine (DF), il se terminait par une phrase analogue, indiquant que Sophie a plus de pouvoir que François. Les participants effectuaient les mêmes tâches que dans l'étude précédente : ils anticipaient les comportements des deux personnages, et expliquaient ces comportements.

L'hypothèse mise à l'épreuve dans cette étude se formule comme suit. La situation DM devrait répliquer les résultats obtenus dans l'étude précédente, amenant les participants à mobiliser fortement les représentations intergroupes de sexe qui justifient la supériorité masculine. En revanche, l'attente concernant la situation DF est plus incertaine. D'une part, étant donné que les traits et comportements masculins sont associés à l'idée même de dominance (e.g., Sidanius et Pratto, 1999), cette situation pourrait conduire à un renversement des perceptions. Sophie, dans la mesure où elle occupe la position de haut statut, sera alors perçue comme plus masculine que François. Mais d'autre part, conformément à l'idée qu'un renversement des rôles sociaux n'est pas suffisant pour annuler l'effet des " différences de sexe enracinées » (ingrained sex differences in personality traits and behavioral tendencies, cf. Eagly et Johnson, 1990), la situation de DF pourrait conduire à l'atténuation de la stéréotypie des deux personnages, c'est-à-dire à une similitude des anticipations fournies pour François et pour Sophie. Ces deux attentes s'expriment en termes d'une interaction entre la situation de domination et le sexe du personnage, que l'analyse des contrastes statistiques permettra de départager. 


\section{Résultats}

Signalons d'emblée que, comme dans l'étude précédente, les corrélations entre comportements anticipés et attributions sont relativement élevées pour les deux personnages $(0,43$ pour Sophie, et 0,59 pour François, $p s<.01)$.

Les scores de comportements anticipés et d'attributions ont été soumis à une ANOVA, dont le plan est 2 (sexe du personnage) x 2 (situation : DM vs. DF) x 2 (sexe du participant), le premier facteur étant en mesure répétée. Le Tableau 3 présente les moyennes du score de comportements anticipés.

Tableau 3 : Score de comportements anticipés $(0=$ féminin ; $1,5=$ autant féminin que masculin ; 3 =masculin). Les écarts-type sont indiqués entre parenthèses. Aucune moyenne ne diffère significativement de 1,5.

\begin{tabular}{cccc}
\hline & \multicolumn{3}{c}{ Situation } \\
\cline { 2 - 3 } & DM & DF & Moyenne \\
\hline François & $1,80(0,77)$ & $1,30(0,99)$ & 1,55 \\
Sophie & $1,15(0,88)$ & $1,85(0,88)$ & 1,50 \\
Moyenne & 1,50 & 1,58 & \\
\hline
\end{tabular}

L'ANOVA ne montre aucun effet du sexe du participant. On constate, comme prédit, une interaction entre la situation et le sexe du personnage, $F(1,36)=5,32, p<.05$. En situation DM, les participants ont tendance à anticiper plus de comportements masculins pour François que pour Sophie, $F(1,36)=3,12, p=.08$. Or en situation $\mathrm{DF}$, on ne remarque aucune différence significative entre les anticipations pour les deux personnages. Concernant François, on assiste à plus d'anticipations de comportements masculins en DM qu'en DF, $F(1,36)=3,16, p=.08$. Au contraire, concernant Sophie, on remarque plus de comportements masculins en DF qu'en $\mathrm{DM}, F(1,36)=6,43, p<.05$. Toutefois, les comparaisons à la valeur centrale de I'indice $(1,5)$ ne font apparaître aucune tendance claire vers la masculinisation ou la féminisation.

L'ANOVA sur le score d'attributions produit, de même que pour les comportements, I'interaction entre la situation et le sexe du personnage, $F(1,36)=13,42, p<.001$. Le Tableau 4 (page suivante) montre les moyennes de ce score.

En situation DM, les comportements de François sont expliqués de manière résolument masculine, tandis que ceux de Sophie sont plutôt féminins, $F(1,36)=10,79$, $p<.005$. De manière spéculaire, en situation DF, les comportements de Sophie sont expliqués de manière résolument masculine, tandis que ceux de François sont jugés plus ambivalents, $F(1,36)=3,59, p=.06$. Ainsi, une position dominante confère à son occupant, qu'il soit homme ou femme, des traits masculins. 
Tableau 4 : Score d'attribution $(0=$ féminin ; $1,5=$ autant féminin que masculin ; $3=$ masculin). Les écarts-type sont indiqués entre parenthèses. Comparaison avec $1,5:{ }^{*} p<.05$, ** $p<.01$.

\begin{tabular}{|c|c|c|c|}
\hline & \multicolumn{2}{|c|}{ Situation } & \multirow[b]{2}{*}{ Moyenne } \\
\hline & DM & DF & \\
\hline François & $2,30 * *(0,86)$ & $1,40(1,19)$ & 1,85 \\
\hline Sophie & $1(1,08)$ & $2,15^{*}(0,99)$ & 1,58 \\
\hline Moyenne & 1,65 & 1,78 & \\
\hline
\end{tabular}

\section{Discussion}

Les résultats de cette étude vont dans le sens de notre hypothèse, tout en la précisant. Une personne est jugée plus masculine lorsque, ayant un meilleur statut professionnel que son partenaire, elle occupe une position dominante dans la hiérarchie professionnelle. Relevons néanmoins que ce phénomène est davantage marqué lorsque les participants expliquent les comportements des personnages au moyen de traits de personnalité que lorsqu'ils leur imputent ces comportements.

Comme attendu, ces résultats reproduisent assez fidèlement ceux obtenus en condition de situation professionnelle de l'Étude 1. Si globalement, et sans surprise, François fait l'objet de descriptions plus masculines que Sophie, la stéréotypisation sexuelle du personnage est sensiblement modulée par son statut social. Un statut élevé - il s'agit en l'occurrence du pouvoir dont dispose la personne sur son partenaire - incite à l'attribution de comportements masculins et à l'explication de ces comportements en termes de stéréotypes masculins. Ce phénomène ne s'atténue que très marginalement lorsque $c^{\prime}$ est la femme qui détient le pouvoir, et non I'homme. En d'autres termes, conformément à un modèle récent des dynamiques identitaires dans les hiérarchies de statuts (Lorenzi-Cioldi, 2002, 2006), la position sociale d'une personne compte davantage que son sexe. L'idée de " différences de sexe enracinées ", qui auraient dû conduire les participants à des perceptions similaires de François et de Sophie en situation de domination de cette dernière, ne peut expliquer les résultats obtenus.

Ces résultats indiquent au contraire que, conformément aux recherches classiques sur la stéréotypie de sexe, I'image de la femme est éloignée de la sphère du pouvoir, cette dernière étant conçue de manière résolument masculine (e.g., Broverman, Broverman, Clarkson, Rosenkrantz et Vogel, 1970 ; Broverman, Vogel, Broverman, Clarkson et Rosenkrantz, 1972 ; Fiske, Bersoff, Borgida, Deaux et Heilman, 1991). Ils suggèrent en outre, de manière cohérente, que lorsqu'une femme accède à un rôle de statut élevé, elle est alors jugée comme plus masculine (e.g., Eagly, Makhijani, et Klonsky, 1992 ; Lorenzi-Cioldi et Buschini, 2005 ; Schein, 1996 ; Schein, Mueller et Jacobson, 1989 ; Twenge, 1997). Et ils aident à comprendre des résultats somme toute surprenants de sondages montrant par exemple que $43 \%$ 
des hommes et $54 \%$ des femmes préfèrent avoir un supérieur hiérarchique de sexe masculin, tandis que seulement $15 \%$ des premiers et $12 \%$ des secondes préfèrent un supérieur de sexe féminin ( $c f$. Swim, Aikin, Hall et Hunter, 1995, p. 200).

Dans leur ensemble, les résultats des deux premières études font émerger une vision androcentrique des hiérarchies de pouvoir. La femme se doit, en quelque sorte, de renier son sexe afin d'accéder à leurs sphères les plus élevées. Or si, dans les sociétés occidentales, les hiérarchies de statuts et de pouvoirs sont censées reposer sur l'attribution de mérite à leurs occupants, la reconnaissance de la discrimination que subissent les membres de certains groupes, comme les femmes précisément, ont amené les milieux dirigeants et politiques à proposer, ici et là, des mesures dites de « discrimination positive » (e.g., Calvès, 2004 ; Harrison, Kravitz, Mayer, Leslie et Lev-Arey, 2006). Ces mesures préférentielles, à caractère volontariste, visent à promouvoir certaines catégories de la population à un domaine (p. ex., la politique) ou à des rôles décisionnaires (p. ex., les postes de cadres) dans lesquels elles sont fortement sous-représentées. Or certains résultats antérieurs portent à croire que, lorsque la femme accède à un poste de haut statut suite à la mise en place d'une mesure positive qui la propulse en tant que femme, elle puisse se juger, et être jugée par autrui, à l'aide de caractéristiques typiquement féminines (LorenziCioldi et Buschini, 2008). Considérons une mesure positive à forte teneur catégorielle, comme la mesure des quotas (Lorenzi-Cioldi, 2002). C'est bien le groupe d'appartenance qui, se sommant aux qualifications requises pour le poste, conduit la postulante à occuper un rôle de cadre dirigeant. Cela devrait donc conduire les participants à conférer plus de féminité, et/ou moins de masculinité, à cette femme. La prochaine étude entendait vérifier cette hypothèse, selon laquelle une promotion avec l'appui d'une politique des quotas s'accompagne de descriptions stéréotypées féminines, plutôt que masculines, de la bénéficiaire.

\section{ÉTUDE 3 : Efficacité professionnelle vs discrimination positive dans la promotion féminine}

\section{Procédure}

Les participants étaient 28 étudiantes et 19 étudiants de 1 ère année de psychologie (âge moyen $=19$ ans). Ils examinaient le scénario décrivant un contexte professionnel identique à celui utilisé dans les études précédentes, mais complété afin d'expliciter les causes de la promotion de Sophie à un poste hiérarchiquement supérieur. En situation de compétence (COMP), le scénario se terminait ainsi : "Pourtant, au départ à la retraite de leur supérieur hiérarchique, c'est Sophie qui est promue et qui prend sa place. En effet, dans le travail, Sophie est beaucoup plus efficace que François ». En situation de discrimination positive (DPOS), la promotion de Sophie était ainsi justifiée : " En effet, face à la rareté des femmes dans les postes d'encadrement, la direction a décidé d'appliquer un quota de femmes. Sophie a donc bénéficié d'une mesure de discrimination positive ». Comme dans les études précédentes, les participants anticipaient les comportements des deux personnages, puis ils les expliquaient. 
Le plan expérimental comporte trois facteurs : 2 (sexe du personnage) $\times 2$ (cause de la promotion : COMP vs. DPOS) x 2 (sexe du participant), le premier facteur étant en mesure répétée. Notre attente est donc que les comportements anticipés et les explications de ces comportements signaleront une perception plus féminine de Sophie dans la condition DPOS que dans la condition COMP. Cette dernière condition devrait quant à elle produire des résultats analogues à ceux observés pour le personnage féminin dans l'Étude 2.

\section{Résultats}

De manière similaire aux études précédentes, les corrélations entre comportements anticipés et attributions sont élevées pour les deux personnages $(0,56$ pour Sophie, et 0,51 pour François, $p s<.01$ ). L'ANOva des comportements anticipés produit d'abord une interaction tendancielle entre le sexe du participant et la cause de la promotion, $F(1,43)=3,69, p=.06$. Les hommes font globalement plus d'anticipations de comportements masculins en DPOS qu'en COMP $(M s=1.78$ et 1.40, respectivement, $F(1,43)=5,17, p<.05)$. Les femmes, quant à elles, anticipent les mêmes comportements dans les deux conditions ( $M s=1,54$ et 1,57 , pour DPOS et pour COMP, respectivement). Cette analyse ne produit pas I'interaction attendue entre le sexe du personnage et la cause de la promotion. Toutefois, une analyse de contrastes planifiés (cf. Rosenthal, Rosnow et Rubin, 2000) indique qu'en condition DPOS, les participants anticipent davantage de comportements masculins pour François que pour Sophie, $F(1,43)=3,22, p=.07$ (voir Tableau 5).

Tableau 5 : Score de comportements anticipés $(0=$ féminin ; 1,5 = autant féminin que masculin ; 3 = masculin). Les écarts-type sont indiqués entre parenthèses. Comparaison avec $1,5: * p<.05$.

\begin{tabular}{cccc}
\hline & \multicolumn{2}{c}{ Cause de la promotion } & \\
\cline { 2 - 3 } & DPOS & COMP & Moyenne \\
\hline François & $1,99 *(0,95)$ & $1,58(1,13)$ & 1,78 \\
Sophie & $1,33(0,78)$ & $1,39(0,88)$ & 1,39 \\
Moyenne & 1,66 & 1,49 & \\
\hline
\end{tabular}

La condition COMP, en revanche, ne met en évidence aucune différence entre Sophie et François. Ici, les descriptions de leurs comportements ne sont teintées d'aucune stéréotypie particulière. Elles sont plus indifférenciés, comme l'indique la proximité du score avec sa valeur centrale $(1,5)$.

L'ANOVA de l'indice d'attributions montre d'abord un effet tendanciel du sexe du participant, $F(1,43)=3,47, p=.06$. Les hommes font plus d'attributions masculines que les femmes ( $M s=1,87$ et 1,64 , respectivement). On remarque aussi un effet de la cause de la promotion, $F(1,43=4,15, p<.05$. Les participants font davantage d'attributions masculines en DPOS qu'en COMP $(M s=1,88$ et 1,63, 
respectivement). On note enfin un effet lié au sexe du personnage, les attributions masculines étant plus prononcées pour François que pour Sophie, $F(1,43)=$ $13,27, p<.001$.

Tableau 6 : Score d'attribution $(0=$ féminin ; $1,5=$ autant féminin que masculin ; $3=$ masculin). Les écarts-type sont indiqués entre parenthèses. Comparaison avec $1,5:{ }^{*} p<.05$, $* * p<.01$.

\begin{tabular}{cccc}
\hline & \multicolumn{2}{c}{ Cause de la promotion } & \\
\cline { 2 - 3 } & DPOS & COMP & Moyenne \\
\hline François & $2,46^{* *}(0,66)$ & $1,88^{*}(0,96)$ & 2,17 \\
Sophie & $1,31(0,96)$ & $1,39(0,85)$ & 1,35 \\
Moyenne & 1,88 & 1,63 & \\
\hline
\end{tabular}

Contrairement à notre attente, les résultats ne font pas apparaître une interaction entre le sexe du personnage et la cause de la promotion. Toutefois, I'analyse des contrastes planifiés met en évidence des effets analogues à ceux observés à propos des comportements. En condition DPOS, les participants font plus d'attributions masculines pour François que pour Sophie, $F(1,43)=12,58, p<.001$, et en condition COMP on n'observe aucune différence entre les attributions pour les deux personnages. Même si François est perçu comme masculin dans les deux conditions, on constate qu'il est jugé davantage masculin en DPOS qu'en COMP, $F(1,43)=6,22, p<.05$. On n'observe en revanche aucune différence significative pour Sophie, dont les descriptions demeurent plus indifférenciées quant aux stéréotypes de sexe.

\section{Discussion}

Les résultats de cette étude ne confirment pas I'hypothèse selon laquelle une femme promue à un poste hiérarchique supérieur en bénéficiant d'une mesure de discrimination positive est jugée plus féminine qu'une femme promue sur la base d'un critère méritocratique, fort estimé dans nos sociétés occidentales. Cependant, ces résultats ne sont pas totalement incompatibles avec cette hypothèse. Dans le contexte de discrimination positive, c'est en fait l'homme qui se révèle être plus masculin que la femme, et non la femme plus féminine que l'homme. Dans la condition de cause méritocratique, il n'y a pas de différence entre les perceptions stéréotypiques des deux personnages. Ces perceptions sont passablement indifférenciées selon les stéréotypes de sexe. Ainsi, la présence de discrimination positive creuse la différence entre les deux personnages, au profit d'une accentuation de la masculinité de François. Au final, le profil des moyennes suggère quelque chose qui s'apparente à notre hypothèse, sans la répercuter entièrement : lorsque la femme est promue grâce à une mesure positive, elle est jugée comme nettement moins masculine que son concurrent homme. En fait, c'est dans cette condition 
que François obtient le score de masculinité le plus élevé pour ce qui est des trois études présentées ici.

Ainsi, les différences observées en présence de discrimination positive proviennent d'une accentuation de la masculinité de l'homme et non pas, comme on l'avait initialement supposé, d'une accentuation de la féminité de la femme. Sans la confirmer, mais sans non plus la contredire, ces résultats modulent sensiblement notre hypothèse. En effet, tout se passe comme si la promotion obtenue par la femme grâce à une mesure positive dispensait celle-ci de se comporter et de se montrer " comme un homme »- sans pour autant acquérir des manières d'être résolument féminines. II n'est donc pas surprenant de constater que la condition DPOS de la présente étude recalque assez fidèlement la condition DM de l'Étude 2 : lorsque Sophie a bénéficié d'une mesure de discrimination positive, et bien que I'on indique qu'elle est dans une position de haut statut, elle est décrite de la même façon que lorsqu'elle est placée en infériorité par rapport à François - elle est moins masculine que lui. Bien que Sophie soit dominante, sa description en termes de faible masculinité par rapport à François persiste si elle a acquis ce statut grâce à une mesure positive.

\section{Discussion générale}

Dans leur ensemble, les résultats issus de la présente recherche illustrent la dynamique des rapports asymétriques entre les sexes, et fournissent quelques indications sur la manière $d^{\prime}$ influer sur ces dynamiques.

La première étude met en évidence que, en l'absence d'indications sur une quelconque différence statutaire entre l'homme et la femme, les participants accentuent la stéréotypie de sexe imputée aux deux personnes dans un contexte professionnel par rapport à un contexte familial ou de couple. Cette différence semble s'appuyer sur l'inférence d'un rapport asymétrique entre l'homme et la femme, en conformité aux croyances concernant les rôles professionnels habituellement assumés par les uns et par les autres ( $c f$. la théorie des rôles sociaux, Diekman et Eagly, 2000 ; Eagly, 1987). Cette explication est corroborée par l'absence de différences significatives entre la condition professionnelle de cette étude et la condition de domination masculine de l'Étude 2. Cela suggère que dans un contexte professionnel mettant en scène un homme et une femme aux diplômes, compétences ou revenus comparables, les participants font néanmoins l'inférence d'un rapport asymétrique conforme aux croyances diffuses sur les rôles habituellement assumés par les hommes et les femmes.

L'Étude 2 met en évidence qu'une position de domination confère à son occupant une stéréotypie masculine. Si ce résultat n'est point surprenant lorsque le personnage est un homme, il est plus intriguant lorsque le personnage est une femme. Tout d'abord, cette "perte de féminité » de la femme qui occupe un rôle dirigeant reproduit, au niveau expérimental, des résultats déjà attestés dans des observations en milieu naturel, où les femmes qui accèdent, par exemple, à des postes de cadres ou de dirigeantes se voient reprocher leurs manières d'être masculines ou androgy- 
nes ( $c f$. Fiske et coll., 1991), voire, plus en profondeur, leur « personnalité » masculine (cf. Schein, Mueller et Jacobson, 1989). Ensuite, I'attribution de masculinité à la femme qui occupe le poste de haut statut signale, conformément aux travaux sur la dominance sociale (e.g., Sidanius et Pratto, 1999), que toute hiérarchie de statuts est associée de manière étroite, sur le plan psychologique, à l'asymétrie des sexes, où l'homme occupe la place du dominant. Les croyances implicites concernant I'inadéquation relative de la femme à occuper des postes de haut statut (cf. Eagly et Johnson, 1990) n'ont apparemment pas été activées par les participants.

La troisième étude était une tentative d'inverser cette dynamique aux apparences néfastes, ou du moins coûteuses sur le plan identitaire, pour les femmes. On faisait en effet l'hypothèse que la femme accédant à un poste de haut statut en bénéficiant d'une mesure positive pourrait dès lors être perçue comme plus féminine, sinon franchement féminine, par rapport à celle qui, évoluant dans un contexte purement méritocratique et plus traditionnel, devait se confronter plus directement à l'homme (cf. Lorenzi-Cioldi et Buschini, 2005). Les résultats n'ont pas corroboré cette hypothèse d'un renversement des perceptions. Toutefois, I'examen plus approfondi de ces mêmes résultats a permis de déceler des tendances compatibles avec notre hypothèse. D'une part, dans la condition de mesure positive, on recense les descriptions les plus masculines de I'homme occupant une position subordonnée que l'on ait relevées dans les trois études. D'autre part, la femme promue grâce à la mesure positive n'est plus franchement masculine (comme elle l'était dans la deuxième étude, en situation de domination). Ainsi, lorsqu'elle accède au statut supérieur grâce à la mesure positive, la femme n'est pas jugée franchement féminine, mais elle n'est pas pour autant jugée masculine (comme elle l'était dans l'Étude 2). De manière cohérente avec notre hypothèse, son « défaut » de masculinité comparativement à l'homme s'accentue dans cette situation.

Relevons enfin que ce n'est que dans cette dernière étude, où il est question de discrimination positive, que l'on voit apparaître des effets liés au sexe du participant. II est possible que cela survienne parce que, dans cette étude, on évoque un enjeu plus structurel pour les individus (i.e., l'accès différencié à la promotion professionnelle), source de rivalité entre les participants des deux sexes. Et ce même s'il ne faut pas oublier que les participants à ces trois expériences étaient des étudiantes et des étudiants relativement jeunes et n'ayant qu'une expérience limitée des contextes professionnels et de leurs spécificités. Afin de répondre à cette limitation, il conviendrait sans doute de répliquer cette recherche avec une population davantage impliquée par des questions de promotion professionnelle, comme par exemple des cadres hommes et femmes en milieu de carrière. Quoi qu'il en soit, le protocole suivi dans le présent travail, nous semble particulièrement bien adapté à l'étude du rôle des représentations intergroupes dans de nombreux contextes sociaux. 


\section{Note}

1. L'ordre de ces jugements n'ayant produit aucun effet, il n'en sera pas tenu compte dans la présentation des résultats.

\section{Bibliographie}

- Abric, J.C. (1976). Jeux, conflits et représentations sociales. Thèse de Doctorat d'Etat de I'université d'Aix en Provence.

- Abric, J.C. (1994). Les représentations sociales : aspects théoriques. In J.C. Abric (Ed.), Pratiques sociales et représentations (pp 11-36). Paris : Presses Universitaires de France.

- Bem, S. L. (1974). The measurement of psychological androgyny. Journal of Consulting and Clinical Psychology, 42, 155-162.

- Bihr, A. et Pfefferkorn, R. (2002). Hommes, femmes, quelle égalité ? Paris : Ed. de l'Atelier.

- Broverman, I. K., Broverman, D. M., Clarkson, F. E., Rosenkrantz, P. S. et Vogel, S. R. (1970). Sex-role stereotypes and clinical judgements of mental health. Journal of Consulting and Clinical Psychology, 34, 1-7.

- Broverman, I. K., Vogel, S. R., Broverman, D. M., Clarkson, F. E. et Rosenkrantz, P. S. (1972). Sex-role stereotypes : a current appraisal. Journal of Social Issues, 28, 59-78.

- Calvès, G. (2004). La discrimination positive. Paris : Presses Universitaires de France.

- Chatard, A., Lorenzi-Cioldi, F. et Guimond, S. (2007). Using Social Dominance Theory to Understand the Interplay Between Relative Group Status and School Achievement. Manuscrit soumis pour publication.

- Deschamps, J.C. (1973). L'attribution, la catégorisation sociale et les représentations intergroupes. Bulletin de Psychologie, 13-14, 710-721.

- Diekman, A. B. et Eagly, A. H. (2000). Stereotypes as dynamic constructs : Women and men of the past, present, and future. Personality and Social Psychology Bulletin, 26, 1171-1188.

- Doise, W. (1969). Stratégies de jeu à l'intérieur, et entre des groupes de nationalité différente. Bulletin du C.E.R.P., 18, 13-26.

- Doise, W. (1973). Rencontres et représentations intergroupes. Archives de Psychologie, 61, 303-320.

- Doise, W., Clémence, A. et Lorenzi-Cioldi, F. (1992). Représentations sociales et analyses de données. Grenoble : Presses Universitaires de Grenoble.

- Doise, W. (2005). Les représentations sociales. In N. Dubois (Ed.), Psychologie sociale de la cognition (pp. 153-207). Paris : Dunod.

- Eagly, A.H. (1987). Sex differences in social behavior : A social role interpretation. London : Lawrence Erlbaum

- Eagly, A. H. et Johnson, B. T. (1990). Gender and leadership style : A meta-analysis. Psychological Bulletin, 108, 233-256.

- Eagly, A. H. et Sczeny, S. (2009). Stereotypes about women, men, and leaders : Have times changed? In M. Barreto, M. K. Ryan, et M. T. Schmitt (Eds), The glass ceiling in the 21st century (pp 21-47). Washington : APA.

- Eagly, A. H., Makhijani, M. G. et Klonsky, B. G. (1992). Gender and the evaluation of leaders: A meta-analysis. Psychological Bulletin, 111, 3-22. 
- Ellemers, N. (2001). Individual upward mobility and the perceived legitimacy of intergroup relations. In J. T. Jost et B. Major (Eds), The psychology of legitimacy (pp. 205-222). New York : Cambridge University Press.

- Fiske, S. T., Bersoff, D. N., Borgida, E., Deaux, K. et Heilman, M. E. (1991). Social science research on trial. Use of sex stereotyping research in Price Waterhouse v. Hopkins. American Psychologist, 46, 1049-1060.

- Flament, C. (2001). Pratiques sociales et dynamique des représentations. In P. Moliner (Ed). La dynamique des représentations sociales (pp. 43-58). Grenoble : Presses Universitaires de Grenoble.

- Harrison, D. A., Kravitz, D. A., Mayer, D. M., Leslie, L. M. et Lev-Arey, D. (2006). Understanding attitudes towards Affirmative Action programs in employment : Summary and meta-analysis of 35 years of research. Journal of Applied Psychology, 91, 1013-1036.

- Hoffman, C. et Hurst, N. (1990). Gender stereotypes : Perception or rationalization? Journal of Personality and Social Psychology, 58, 197-208.

- Jost, J.T. et Banaji, M.R. (1994). The role of stereotyping in system-justification and the production of false consciousness. British Journal of Social Psychology, 33, 1-27.

- Jost, J. T., Banaji, M. R. et Nosek, B. A. (2004). A decade of system justification theory : Accumulated evidence of conscious and unconscious bolstering of the status quo. Political Psychology, 25, 881-919.

- Jost, J. T. et Burgess, D. (2000). Attitudinal ambivalence and the conflict between group and system justification motives in low status groups. Personality and Social Pychology Bulletin, 26, 293-305.

- Jost, J. T. et Kay, A. C. (2005). Exposure to benevolent sexism and complementary gender stereotypes : Consequences for specific and diffuse forms of system justification. Journal of Personality and Social Psychology, 88, 498-509

- Kelley, H. H. (1992). Common-sense psychology and scientific psychology. Annual Review of Psychology, 43, 1-24.

- Lorenzi-Cioldi, F. (1994). Les androgynes. Paris : Presses Universitaires de France.

- Lorenzi-Cioldi, F. (2002). Les représentations des groupes dominants et dominés. Collections et agrégats. Grenoble : Presses Universitaires de Grenoble.

- Lorenzi-Cioldi, F. (2006). Group status and individual differentiation. In T. Postmes et J. Jetten (Eds), Individuality and the group : Advances in social identity (pp. 93-115). London : Sage.

- Lorenzi-Cioldi, F. et Buschini, F. (2005). Vaut-il mieux être une femme qualifiée ou être qualifiée de femme? Effets paradoxaux de la catégorisation dans la discrimination positive. In M. Sanchez-Mazas et L. Licata (Eds), L'autre. Regards psychosociaux (pp. 279-308). Grenoble : Presses Universitaires de Grenoble.

- Lorenzi-Cioldi, F., et Buschini, F. (2008). When the Glass Ceiling Collapses, Walls are Erected: Positive and Negative Consequences of Psychological Essentialism in Affirmative Action. In : S. Badaloni, C.A. Drace, O. Gia, C. Levorato, et F. Vidotto (Eds), Under-representation of women in science and technology (pp. 27-40). Padova : Cleup.

- Meron, M., Okba, M. et Viney, X. (2006). Les femmes et les métiers : vingt ans d'évolutions contrastées. Données sociales, Insee.

- Moliner, P. et Gutermann M. (2004). Dynamique des descriptions et des explications dans une représentation sociale. Papers On Social Representations, 13, 1-12.

- Moscovici, S. (1961). La psychanalyse, son image, son public. Paris : Presses Universitaires de France. 
- Ridgeway, C. L. et Erickson, K. G. (2000). Creating and spreading status beliefs. American Journal of Sociology, 106, 579-615.

- Rosenthal, R., Rosnow, R. et Rubin, D. B. (2000). Contrasts and effect sizes in behavioral research : A correlational approach. New York : Cambridge University Press.

- Schein, V. E. (1996). Thin manager-think male : A global phenomenon? Journal of Organizational Behavior, 17, 33-41.

-Schein, V. E., Mueller, R. et Jacobson, C. (1989). The relationship between sex role stereotypes and requisite management characteristics among College students. Sex Roles, 20, 103-110.

- Sidanius, J. et Pratto, F. (1999). Social dominance : An intergroup theory of social hierarchy and oppression. New York : Cambridge University Press.

- Swim, J. K., Aikin, K. J., Hall, W. S. et Hunter, B. A. (1995). Sexism and racism : Old-fashioned and modern prejudices. Journal of Personality and Social Psychology, 68, 199-214.

- Tajfel, H. (1972). La catégorisation sociale. In S. Moscovici (Ed), Introduction à la psychologie sociale (pp. 273-302). Paris : Larousse.

- Tajfel, H. et Wilkes, H. (1963). Classification and quantitative judgment. British Journal of Psychology, 54, 101-114.

- Twenge, J. M. (1997). Changes in masculine and feminine traits over time : A meta-analysis. Sex Roles, 36, 305-325.

- Vidal, J. (2003). Noyau central et stéréotypie : la question de la durabilité du changement. Cahiers Internationaux de Psychologie Sociale, 60, 50-58.

- Vinet, E. et Moliner, P. (2006). Asymétries de la fonction explicative des représentations intergroupes hommes/femmes. Cahiers Internationaux de Psychologie sociale, 69, 47-57. 